\title{
Application of Fibre Optic Range-Resolved Interferometric Vibrometry to a Full-Scale Feathered Propeller in a Wind Tunnel
}

\author{
Lawson N.J., Kissinger T., Finnis M.V. *, James S.W., Tatam R.P. \\ School of Aerospace, Transport and Manufacturing \\ Cranfield University \\ Cranfield MK43 OAL \\ U.K. \\ *Defence Academy, Cranfield University, Shrivenham, Wilts SN6 8LA. U.K. \\ n.lawson@cranfield.ac.uk \\ +44-1234-758245
}

\begin{abstract}
:
The vibration characteristics of a full scale Jetstream 31 turboprop propeller blade are analysed using a fibre optic range-resolved vibrometer. Using this vibrometer, a $1.2 \mathrm{~m}$ length propeller blade was interrogated on the low pressure surface at selected spanwise positions. The spectral characteristics of the surface were recorded over wind tunnel speeds ranging from $5 \mathrm{~m} / \mathrm{s}$ to $30 \mathrm{~m} / \mathrm{s}$ in an attempt to decouple an expected aerodynamic shedding frequency from the natural structural frequency of the blade. However, the dominant frequency was found to be the natural frequency and no evidence of shedding aerodynamic frequencies were found using subsequent hot-wire anemometry measurements. Further work is planned to isolate structural and aerodynamic effects, including measurements adjacent to the aerofoil surface and a computation model.
\end{abstract}

Key words: laser vibrometer, hot-wire anemometer, propeller aerodynamics.

\section{Introduction}

The aerodynamics of aircraft propellers appears very mature, with the first designs dating back to the early 1900's [1]. One of the most significant developments in this field was the hydromatic propeller in 1938 which allowed the pitch of the propeller to be finely controlled in order to set engine speed at any flight speed [2]. This control system also allowed an arrangement known as feathering when, under engine failure conditions, all the propeller blades on the hub are rotated $90^{\circ}$ to the flow to minimize drag (See Figure 1).

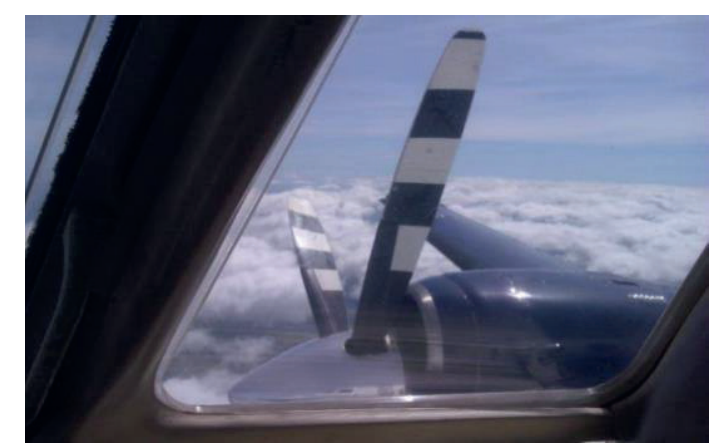

Fig.1. Feathered propeller configuration
Little detailed aerodynamic work has been published on this feathered configuration, as manufacturers own the design data. When feathered at low flight speeds, however, it has been observed that the blades vibrate [3], which implies potentially high blade drag and structural interaction of the blades with the aerodynamics. This paper applies an advanced optical technique, a fibre optic range-resolved vibrometer $[4,5]$, with traditional measurement methods, including hot wire anemometry [6] and surface flow visualization, to establish whether there is an interaction of the aerodynamics of a feathered propeller blade with the structure of the blade.

\section{Experimental Rig}

The experimental rig was based on a full sized, $1.2 \mathrm{~m}$ length production Dowty Jetstream 31 propeller blade, mounted in a $2 \mathrm{~m}$ open jet wind tunnel (see Figure 2). The blade was held on a milling machine chuck at the blade hub to allow twist and tilt of the blade so that appropriate relative wind conditions could be obtained. In these experiments, a set of spanwise pitch, $\phi$, and angle of attack, $\alpha$, conditions (see Figure 3 ) were tested over a range of wind tunnel speeds 
varying from $5 \mathrm{~m} / \mathrm{s}$ to $30 \mathrm{~m} / \mathrm{s}$. The pitch angle $\phi$ was based on flight test data [3] and ranged from $3.3^{\circ}$ to $9.5^{\circ}$. The angle of attack was fixed at $5^{\circ}$. Both the pitch angle $\phi$ and angle of attack $\alpha$ were set using a reference line, obtained by insertion of a bar into a hub datum hole. This datum system is used by aircraft engineers to set the blade angles up on actual aircraft. At $30 \mathrm{~m} / \mathrm{s}$, the tunnel achieved a maximum Reynolds number condition of around $50 \%$ of the flight Reynolds number. As will be outlined in the following section, initial flow visualization tests showed the blade to be insensitive to Reynolds number and so it was assumed the general characteristics of the blade under these conditions were representative of flight conditions.

The wind tunnel was controlled through a serial interface to the motor and achieved stable tunnel speeds, typically within $0.1 \mathrm{~m} / \mathrm{s}$. Centreline tunnel turbulence intensity was less than $0.5 \%$ and the propeller blade was mounted in the centre of the jet, with the hub positioned just on the edge of the jet (see Figure 2).

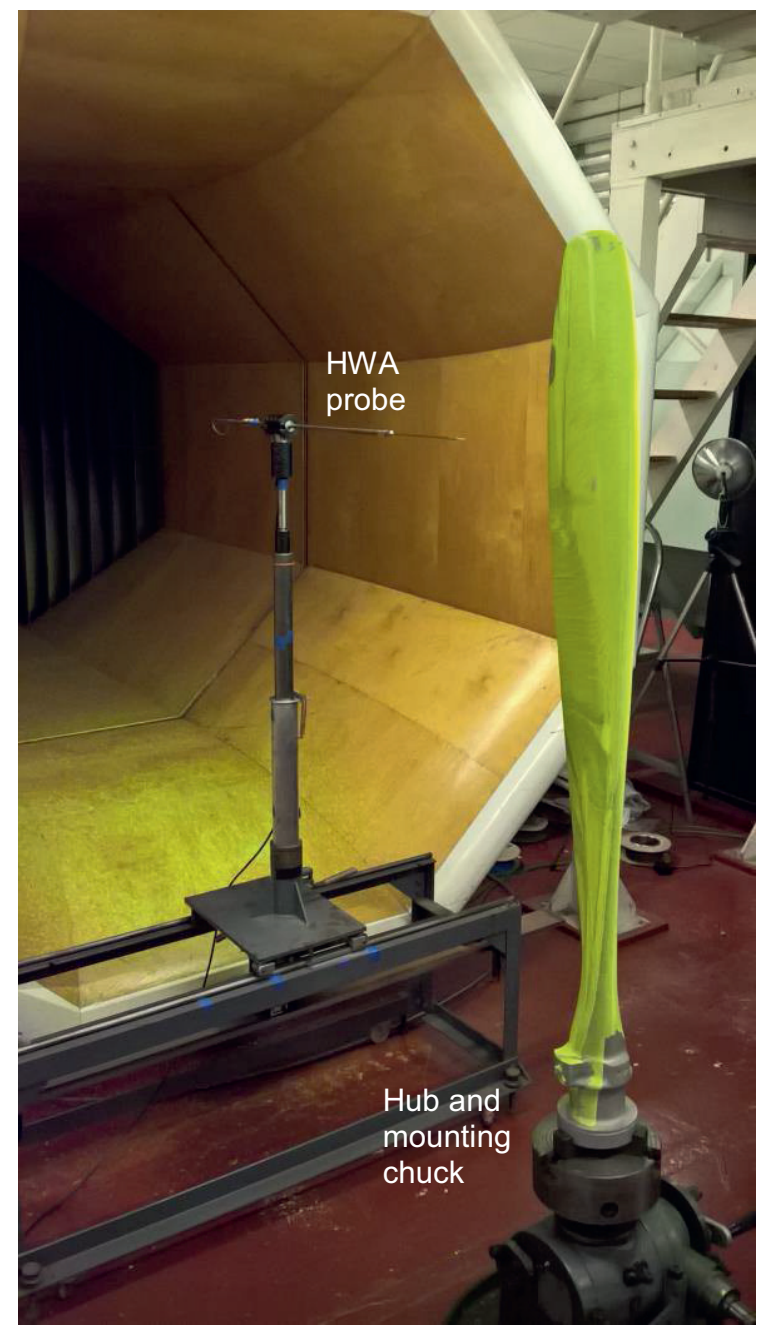

Fig.2.Wind tunnel set-up with hot wire anemometer

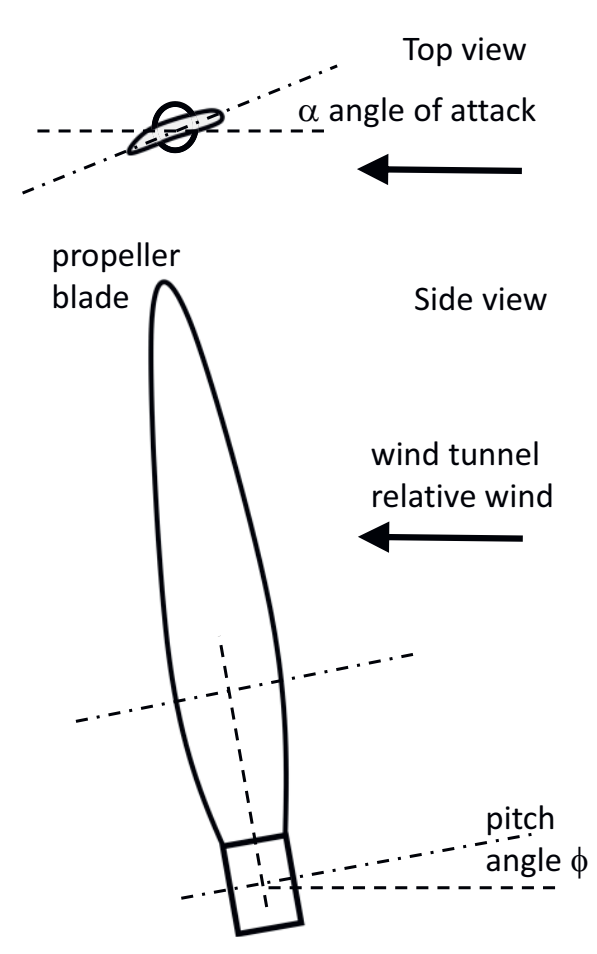

Fig.3.Wind tunnel propeller setting angles

\section{Surface Flow Visualisation}

In order to establish the general flow characteristics around the propeller blade, initial tests were completed using surface flow visualization. Through a range of test conditions, the blade was first coated with an oleic oil and fluorescent pigment emulsion. The wind tunnel was then run at a fixed condition until the flow pattern stabilized and the emulsion dried on the propeller surface. Images were then taken of the resulting flow pattern using a digital camera, while the blade was illuminated by ultra-violet light. A typical black and white image with key flow features is shown in Figure 4.

The result shows the surface flow to be highly three dimensional, with a number of key features including a separation line towards the top half of the propeller, which is confined by a region of attached flow in the middle section of the blade. Below this attached region the flow has even greater 3D complexity, including a node and a further separation line that extends to the hub at the bottom of the blade.

To establish the sensitivity of the flow features to Reynolds number, the tests were repeated at wind speeds of $15 \mathrm{~m} / \mathrm{s}, 20 \mathrm{~m} / \mathrm{s}$ and $25 \mathrm{~m} / \mathrm{s}$ at fixed pitch angles and angles of attack. Results showed the general surface flow features to be consistent across these test conditions. 
Changes in pitch angle also showed no significant change in the general flow features and, therefore, it was concluded at this stage of testing that increases in Reynolds number or pitch did not appear to have a major effect on the flow structure.

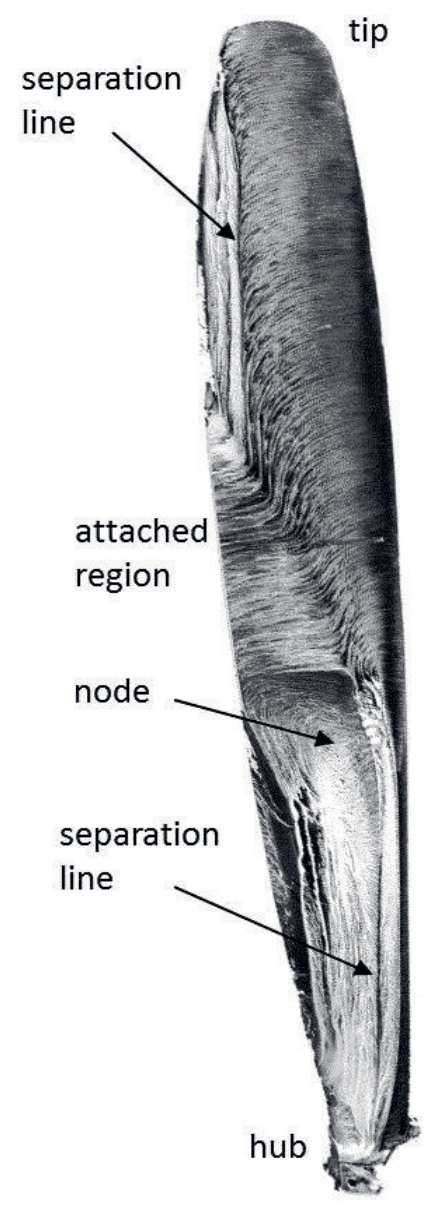

Fig.4.Surface flow visualization $\left(\phi=7^{\circ}, \alpha=5^{\circ}\right.$, $25 \mathrm{~m} / \mathrm{s}$ )

\section{Range Resolved Fibre Optic Vibrometer Measurements}

Using the flow visualization results, an area was selected in the top section of the blade, near the separation line, to examine the vibration characteristics of the propeller blade using a fibre optic, range-resolved vibrometer. At this stage, the study was expected to establish a relationship between wind tunnel speed and shedding frequency off the rear section of the blade tip. Previous work on 2D aerofoils has suggested that a distinct shedding frequency may exist, which varies with tunnel speed, consistent with a fixed Strouhal number $[7,8]$. Therefore if the shedding frequency has a dominant effect on the blade structure, vibrometer measurements would show a strong frequency behavior that matches the aerodynamic Strouhal number.

The fibre optic range-resolved vibrometer is based on a $1551 \mathrm{~nm}$ laser diode source with beam modulation and demodulation carried out using a low cost programmable gate array (FPGA). Fresnel fibre tip reflection is used as an interference reference source, providing a system with a compact sensor head and with complete downlead insensitivity. This system provides an estimated resolution of $5 \mathrm{~nm}$ at a $21 \mathrm{kHz}$ bandwidth, in this case with the sample rate of $49 \mathrm{kHz}$, downsampled to $1.5 \mathrm{kHz}[4,5]$. Figure 5 and 6 shows the basic vibrometer setup used for these tests.

(a) Optical \& Experimental Setup

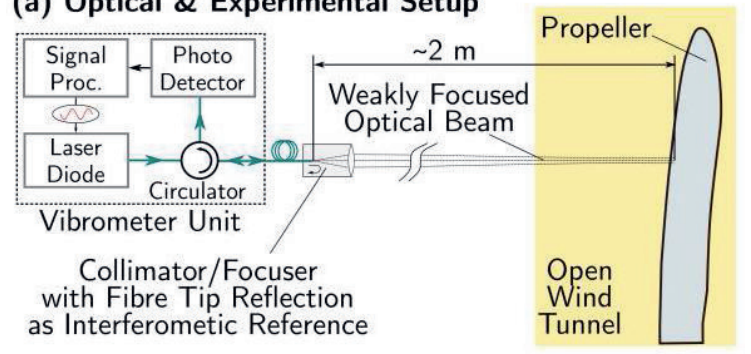

(b) Fully Enclosed Vibrometer Unit

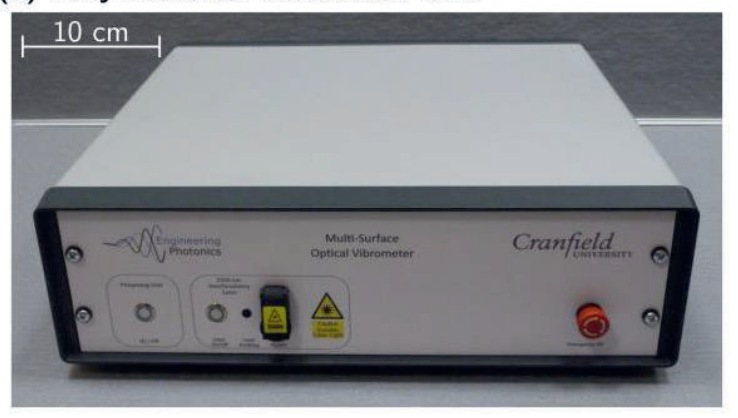

Fig.5.Basic schematic of the fibre optic rangeresolved vibrometer set-up

Spectral plots of the data obtained from the propeller over a range of wind tunnel speeds from $15 \mathrm{~m} / \mathrm{s}$ to $25 \mathrm{~m} / \mathrm{s}$ are shown in Figures 7 and 8 . The contour plot in Figure 8 illustrates the spectra over the full tunnel speed range. The results show a consistent spectrum with a dominant peak at a frequency of around $23 \mathrm{~Hz}$. Further energy in the spectrum is found in the region of $35 \mathrm{~Hz}, 50 \mathrm{~Hz}, 65 \mathrm{~Hz}$ and $90 \mathrm{~Hz}$. However, the general characteristics of the spectra do not change with wind tunnel speed, which suggests that there is no influence of an aerodynamic characteristic on the blade structure. If there was such an aerodynamic driver, the spectral peaks would be expected to shift proportionally with wind tunnel speed.

A further experiment was undertaken with the wind tunnel turned off by pinging the blade to obtain the natural frequency of the structure and to record the spectrum with the vibrometer. 
In this case, the natural frequency matched precisely the $23 \mathrm{~Hz}$ dominant frequency seen in the spectra in Figure 7. Further decomposition of the spectral data using wavelet methods did not lead to any additional evidence of an aerodynamic influence on the blade. Therefore it was concluded, at this stage, that the blade's structural behaviour was independent of the aerodynamic flow over the blade.

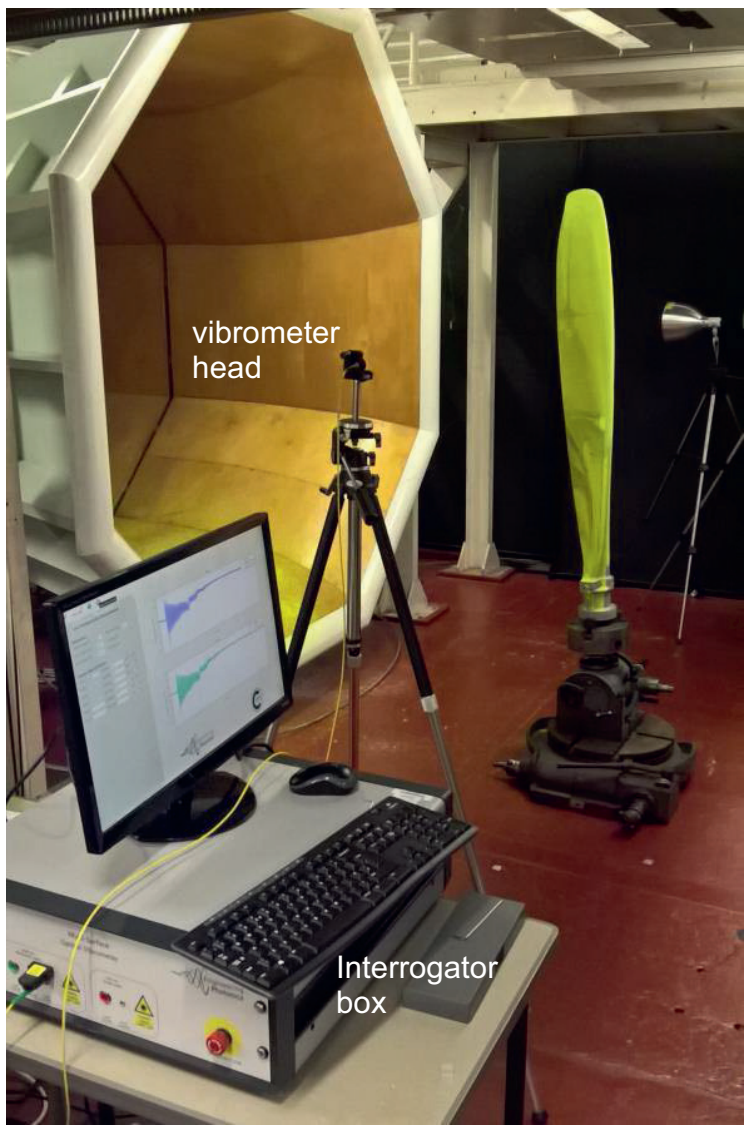

Fig.6.Fibre optic range-resolved vibrometer set-up in the wind tunnel

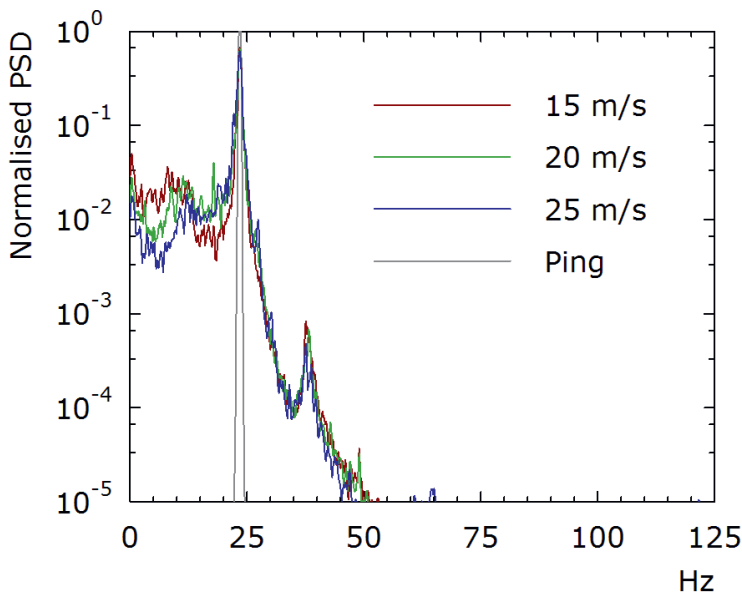

Fig.7. Vibration spectra obtained using the fibre optic range-resolved vibrometer $(333 \mathrm{~mm}$ from tip, $70 \mathrm{~mm}$ from leading edge, ping is natural frequency)

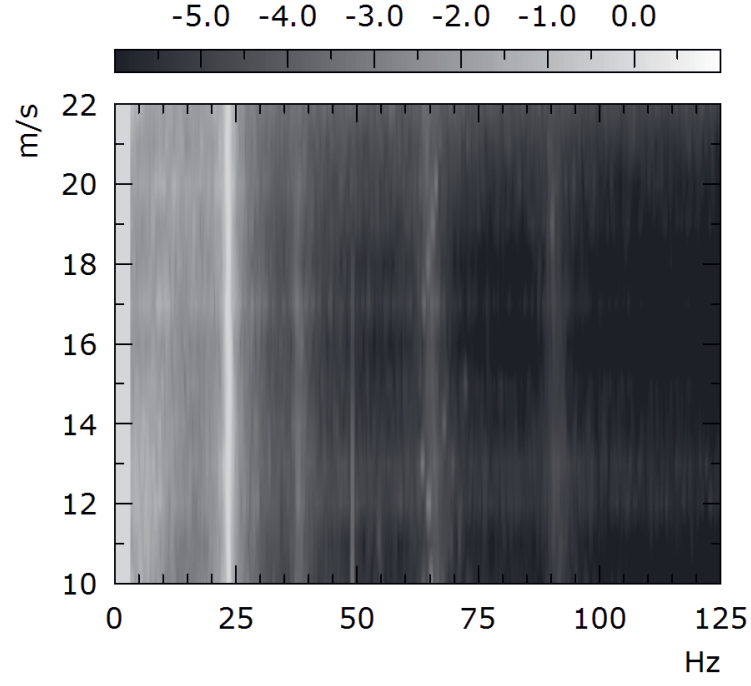

Fig. 8. Contour plot of spectra of data obtained using the fibre optic range-resolved

\section{Hot Wire Anemometry Measurements}

To investigate whether an aerodynamic shedding characteristic was present on the propeller blade, one dimensional hot wire anemometry (HWA) measurements were taken adjacent to the trailing edge at the mid-span position. The HWA set-up consisted of a Dantec 55P05 gold plated probe mounted on a Dantec $55 \mathrm{H} 22$ probe support and connected to a Dantec 56C01/56C17 CTA / bridge combination with a $4 \mathrm{~m}$ probe cable (see Figure 2). The bridge output was measured using a Dewetron signal-processing voltage module connected to a National Instruments 6036E 16 bit $A / D$ convertor. The Dewetron voltage module was set with its low pass filter at $1 \mathrm{kHz}$. Sampling was at $2 \mathrm{kHz}$ for typically $30 \mathrm{~s}$ and the wire was mounted in a vertical position to capture any streamwise wake fluctuations.

Horizontal traverses were initially completed to capture the mid-point of the wake, as shown in Figure 9. The spectra were then examined at the mid-point, as shown in Figure 10, for two wind tunnel speeds.

One of the wind tunnel speeds chosen was lower than the range tested for the vibrometer and the surface flow visualization, as previous work has reported changes in shedding characteristics at the lower Reynolds number range $[7,8]$, corresponding in this case to a wind speed of $5 \mathrm{~m} / \mathrm{s}$. However if the spectra are examined for both speeds, there is no significant change with tunnel speed and there is no dominant frequency at any of the spectral peaks found in the vibrometry measurements, for example the $23 \mathrm{~Hz}$ mode. 


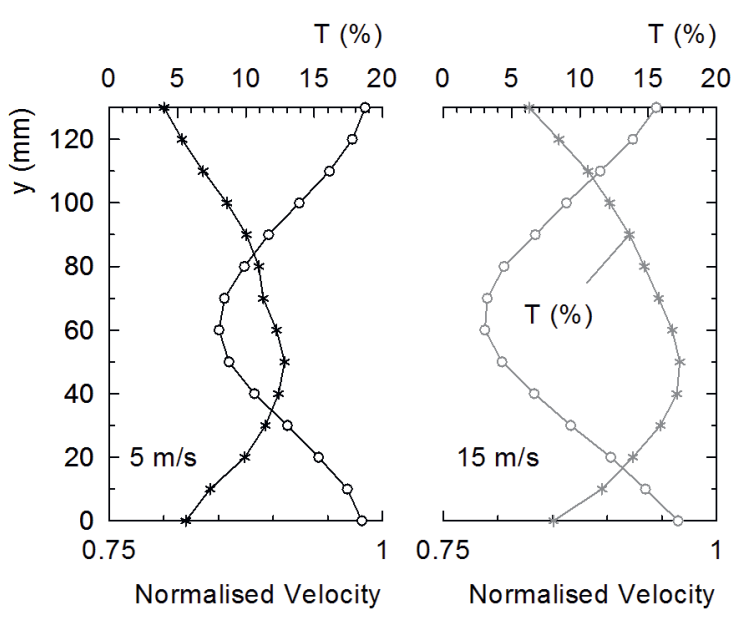

Fig. 9.HWA wake data at a mid-span, trailing edge position

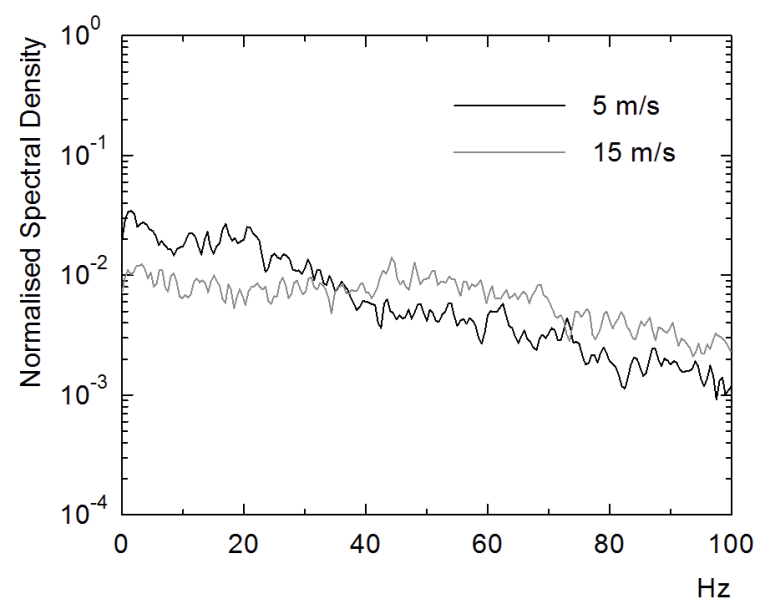

Fig.10.HWA wake spectra at a mid-span, trailing edge position (mid-point wake position, T(\%) turbulence intensity)

\section{Discussion}

The initial motivation for the measurements was to see if it was possible to decouple aerodynamic and structural frequencies by using the high sensitivity of the vibrometer. In a stalled aerofoil, shedding frequencies are often generated and generally follow well-presented characteristic Strouhal numbers. However, in all the measurements made here, although the surface flow visualization indicated separation regions on the aerofoil, the vibrometer consistently showed that the structural frequency of the blade to dominated the results, without any evidence of aerodynamic shedding.

In an attempt to isolate this finding, given that the wake behind an aerofoil contains information on the flow upstream on the upper surface of the aerofoil, HWA measurements where then made at an area downstream of the blade which was thought to have the highest probability of separated flow. However, these results still did not allow the identification of any dominant shedding frequencies, leading to the conclusion that, although the flow is stimulating the natural frequency of the blade, any local separation on the upper surface potentially leads to reattachment before leaving the leading edge of the blade. This effect would then both cause the blade to vibrate, but would also suppress significant fluctuations in the wake.

Hence future work could include more local HWA measurements adjacent to the top surface of the blade to confirm the reattachment phenomena. Alternatively, the vibrometer may be adapted for point wise measurements of density gradient near the trailing edge to interrogate the flow optically, particularly at the higher wind tunnel speeds. This may allow a more detailed investigation of both structural and aerodynamic frequencies. A further investigation may also include the use of a flow diagnostic method such as particle image velocimetry (PIV) [9] in selected regions, to examine wake structure or pressure side blade structure.

\section{Conclusions}

A fibre optic range-resolved vibrometer has been used to measure the vibration characteristics of a full scale Jetstream 31 propeller blade in a feathered position in a low speed wind tunnel. The measurements were made in an attempt to isolate the structural modes of the blade from the expected aerodynamic shedding frequencies, usually seen on a stalled aerofoil. However, although surface flow visualization indicated areas of separation on the aerofoil, the vibrometer spectra did not reveal any aerodynamic frequencies and subsequent hot-wire anemometry measurement also could not find any aerodynamic shedding characteristics.

Further work is now planned to interrogate the flow near the aerofoil surface and to develop an unsteady computational model to isolate any potential unsteady flow effects. The fibre optic vibrometer may also be adapted to measure density gradients, allowing more in-depth spectral characterization.

\section{Acknowledgements}

The authors would like to acknowledge the Cranfield University workshop staff at Shrivenham Defence Academy.

\section{References}

[1] H.C. Watts, The Design of Screw Propellers: With Special Reference to Their Adaptation for Aircraft, Longmans (1920) 
[2] American Sociery of Mechanical Engineers, Historic Mechanical Engineering Landmarks, Hydromatic propeller, web reference https://www.asme.org/about-asme/who-weare/engineering-history/landmarks/149hydromatic-propeller, accessed April 2018

[3] H. Jacques, Simulation of Propeller Effects on the Jetstream 31 Aircraft, Cranfield University, MSc Thesis (2014)

[4] T. Kissinger, T.O.H. Charrett, and R.P Tatam, Range-resolved interferometric signal processing using sinusoidal optical frequency modulation, Opt. Express 23(7), 9415-9431 (2015); doi: 10.1364/OE.23.009415

[5] T. Kissinger, T.O.H. Charrett, S.W, James, A. Adams, A. Twin and R.P. Tatam. Simultaneous laser vibrometry on multiple surfaces with a single beam system using range-resolved interferometry, In Proc. SPIE, vol. 9525, p. 952520 (2015); doi: 10.1117/12.2183281

[6] H.H. Brun. Hot-Wire Anemometry: Principles and Signal Analysis, OUP Oxford (4 May 1995), ISBN 978-0198563426.

[7] S Yarusevych, SH Boutilier, Vortex Shedding of an Airfoil at Low Reynolds, AIAA Journal 49(10), 2221-2227 (2011); doi: 10.2514/1.J051028

[8] K.B.M.Q. Zaman, D.J. McKinzie, C.L. Rumsey, A natural low-frequency oscillation of the flow over an airfoil near stalling conditions, J.Fluid Mech. 202, 403-442 (1989); doi: $10.1017 / \mathrm{S} 0022112089001230$

[9] M Raffel, C.E. Willert, S. Wereley, J. Kompenhans, Particle Image Velocimetry: A Practical Guide, Springer Berlin (2007) 